\title{
Papers
}

\section{Effects of locality based community hospital care on independence in older people needing rehabilitation: randomised controlled trial}

\author{
John Green, John Young, Anne Forster, Karen Mallinder, Sue Bogle, Karin Lowson, Neil Small
}

\begin{abstract}
Objective To determine the effects on independence in older people needing rehabilitation in a locality based community hospital compared with care on a ward for elderly people in a district general hospital.

Design Randomised controlled trial.

Setting Care in a community hospital and district general hospital in Bradford, England.

Participants 220 patients needing rehabilitation after an acute illness that required hospital admission.

Interventions Patients were randomly allocated to a locality based community hospital or to remain within a department for the care of elderly people in a district general hospital. Main outcome measures Primary outcomes were Nottingham extended activities of daily living scale and general health questionnaire 28 (carer). Secondary outcomes were activities of daily living (Barthel index), Nottingham health profile, hospital anxiety and depression scale, mortality, destination after discharge, satisfaction with services, carer strain index, and carer's satisfaction with services.

Results The median length of stay was 15 days for both the community hospital and the district general hospital groups (interquartile range: community hospital 9-25 days; district general hospital 9-24 days). Independence at six months was greater in the community hospital group (adjusted mean difference $5.30,95 \%$ confidence interval 0.64 to 9.96). Results for the secondary outcome measures, including care satisfaction and measures of carer burden, were similar for both groups. Conclusions Care in a locality based community hospital is associated with greater independence for older people than care in wards for elderly people in a district general hospital.
\end{abstract}

\section{Introduction}

Community hospitals, loosely defined as small hospitals with few onsite diagnostic facilities or specialised services, ${ }^{1}$ are a long established but contentious ${ }^{2}$ component of healthcare provision. Interest in community hospitals in England has been renewed, stimulated by the contraction of the district general hospital and the shift towards locality based health services under the umbrella term intermediate care. ${ }^{3}$ This revival, however, is more an opportunistic development than an evidence based one. ${ }^{14}$ Few reports describe evaluations of community hospitals and these are unconvincing, being dominated by considerations of resources rather than clinical outcomes. ${ }^{1}$ A community hospital can result in lower bed usage in a district general hospital, but overall bed utilisation (district general hospital and community hospital combined) may be higher. ${ }^{5-7}$ This increased hospital stay may be acceptable if the overall quality of care is improved such that patient satisfaction and clinical outcomes are superior. To date, no studies have tackled these issues. We assessed the effectiveness of a locality based community hospital on independence in older people needing rehabilitation.

\section{Participants and methods}

Our study was based in a department dealing with the care of elderly people in a metropolitan city with a population of about 400000 people. The department has five wards in a district general hospital and receives emergency admissions from casualty and general practitioners for patients aged over 76 years with acute medical conditions. The wards provide multidisciplinary care, with nurses, therapists, dieticians, and pharmacists led by 10 geriatricians in accordance with described good practice. ${ }^{8}$

The community hospital we studied opened in 1996 and provides locality based care for predominantly older patients who reside in one of the three primary care trusts in Bradford city (population 92 272; 17\% over 65). The hospital has strong links to local general practitioners and local community health and social services (see table A on bmj.com).

\section{Protocol}

Patients were eligible for the trial if they were registered with a general practitioner in the primary care trust served by the community hospital and were considered by the responsible geriatrician to be medically stable and in need of post-acute rehabilitation care. We excluded patients if they had features of medical instability (pyrexia, breathlessness at rest, history of chest pain within the previous 48 hours, or need for intravenous drugs $)^{10}$; were drowsy or unconscious; required specialist rehabilitation in the stroke unit, or treatment in other departments such as surgery or coronary care; or needed new placement in a care home. Patients and their carers gave written informed consent or assent was sought from a carer or relative when patients had impaired capacity.

Baseline assessment by a research nurse included reason for admission, current input from social care services, cognitive function (abbreviated mental test score), ${ }^{11}$ activity restriction before admission (Nottingham extended activities of daily living and Barthel index), ${ }^{12-14}$ and other outcome measures (see bmj.com).

Additional information is on bmi.com 


\section{Assignment}

We stratified patients by cognitive impairment using the abbreviated mental test (categories $0-7 ; 8-10$ ) and by activity restriction using the baseline Barthel index score (categories 0-13; 14-20). Randomisation to community hospital (intervention) or usual care (control) was by numbered, sealed, opaque envelopes prepared from random numbers tables, which initially used four length permuted blocks in a ratio of one intervention group to each control group. Owing to pressures on beds in the district general hospital, we changed the randomisation schedule after two months (26 patients had been recruited) to three length permuted blocks in a ratio of two intervention groups to each control group. We used short length blocks to minimise the potential for disruption to clinical services. Randomisation was carried out away from the clinical setting and, as patients were recruited from several wards, the ward staff were blinded to randomisation. The research nurse responsible for recruitment was unaware of the stratification boundaries and block length. We aimed to transfer patients randomised to the community hospital group within two days in accord with existing local practice.

\section{Intervention group}

Patients allocated to community hospital care were assessed by the multidisciplinary team and received an individual care plan designed to maximise recovery and promote independence. Home assessments before discharge are commonly arranged, and discharge is coordinated with the local social service team. The consultant visited the hospital at least twice a week and the hospital practitioner visited the hospital each weekday. Local general practitioners provided out of hours cover (see bmj.com for input from nursing and therapy teams).

\section{Control group}

Usual care consisted primarily of an extended stay in the care of the elderly ward in the local district general hospital but could include transfer to a non-locality based community hospital or to social service facilities, both of which offered slow track rehabilitation.

\section{Outcome measures, follow up, and blinding}

For the primary outcome measures we used the Nottingham extended activities of daily living scale for patients, a valid and reliable measure, ${ }^{12}{ }^{15}$ and the general health questionnaire 28 for carers. ${ }^{16}$ Other outcome measures are listed in table B on bmj.com. Information on death, length of stay in hospital, and destination after discharge was collected.

Follow-up assessments were at one week after hospital discharge, and at three and six months after recruitment.

A researcher who had not been involved with recruitment visited the patients at home and completed the outcome measures. Unblinding of patient allocation was recorded and the researcher stated the perceived trial group for each patient at the final assessment. We measured agreement between perceived and actual allocation using the $\kappa$ statistic.

\section{Statistical analysis}

We defined a clinically meaningful difference as a two point improvement of the Nottingham extended activities of daily living scale (0-22 scoring). We used the revised 0-66 scoring in accord with recent practice. For our sample size calculation we used a standard deviation of 5.3 recently obtained from an intermediate care study of over 300 frail older people. ${ }^{22}$ This method indicated a sample size of 400 for $1 \%$ significance and $85 \%$ power, and 240 for $5 \%$ significance and $85 \%$ power. We planned to recruit 500 patients to allow for dropouts.
Table 1 Baseline characteristics of patients and lengths of stay. Values are numbers (percentages) unless stated otherwise

\begin{tabular}{lcc} 
Characteristics & $\begin{array}{c}\text { Community hospital group } \\
(\mathbf{n}=\mathbf{1 4 1})\end{array}$ & $\begin{array}{c}\text { District general hospital } \\
\text { group }(\mathbf{n}=\mathbf{7 9 )}\end{array}$ \\
\hline Women & $109(77)$ & $59(75)$ \\
\hline Men & $32(23)$ & $20(25)$ \\
\hline Mean (SD) age (years) & $86.3(5.7)$ & $85.5(6.0)$ \\
\hline Care status: & & $52(66)$ \\
\hline Lives alone & $97(69)$ & $22(28)$ \\
\hline Lives with carer & $37(26)$ & $5(6)$ \\
\hline Lives in care & $7(5)$ &
\end{tabular}

Received social care before

admission:

\begin{tabular}{lcc}
\hline Yes & $66(47)$ & $35(44)$ \\
\hline No & $68(48)$ & $39(49)$ \\
\hline In care & $7(5)$ & $5(6)$ \\
\hline $\begin{array}{l}\text { Median (interquartile range) } \\
\text { pre-admission Barthel } \\
\text { index score }\end{array}$ & $18(16-19)$ & $18(16-19)$ \\
\hline
\end{tabular}

Abbreviated mental test

scores:

\begin{tabular}{lcc}
\hline $0-7$ & $37(26)$ & $22(28)$ \\
\hline $8-10$ & $104(74)$ & $57(72)$
\end{tabular}

Median length (range;

interquartile range) of

hospital stay:

\begin{tabular}{lcc}
\hline $\begin{array}{l}\text { Admission to } \\
\text { randomisation }\end{array}$ & $6(0-63 ; 3-10)$ & $5(0-31 ; 3-8)$ \\
\hline $\begin{array}{l}\text { Randomisation to } \\
\text { discharge }\end{array}$ & $15(2-106 ; 9-25)$ & $15(1-194 ; 9-24)$ \\
\hline Community hospital $^{*}$ & $14(1-105 ; 9-24)$ & - \\
\hline
\end{tabular}

*Based on 121 patients.

Our primary analysis was an intention to treat comparison of change in scores on the Nottingham extended activities of daily living scale from baseline to six months between the intervention and control groups. We used unpaired $t$ test and the Mann-Whitney U test for unadjusted values. We also carried out an adjusted intention to treat comparison using analysis of covariance to adjust for the baseline variables of age, sex, institutional care, mental status, and baseline Barthel index score. To allow for potential bias due to the exclusion of patients who had died, we used the Mann-Whitney U test to compare the groups for the change in Nottingham extended activities of daily living scale scores from preadmission to the six month assessment after recoding the six month scores for these patients as zero (worst outcome). The primary outcome for carers was the difference between the groups for change in general health questionnaire 28 scores between baseline and six months using the Mann-Whitney U test.

For our secondary analyses we used the Mann-Whitney U test to compare the groups for changes in the total Nottingham extended activities of daily living scale scores and general health questionnaire 28 scores between baseline and the follow-up assessments at one week and three months. We carried out similar analyses for each assessment point for the subsections of the Nottingham health profile, the total Barthel index score, and the anxiety and depression subsections of the hospital anxiety and depression scale. We used the Mann-Whitney U test to examine the differences between the groups for the carer strain index scores at the follow-up assessments at one week and three and six months. The $\chi^{2}$ test was used to examine differences in mortality and destination after discharge between the groups. We created a dichotomous response (satisfied or not satisfied) for patient and carer satisfaction scores, and we compared the groups using the $\chi^{2}$ test. 


\section{Results}

Of the 2232 potential participants identified between November 2000 and September 2002, 266 remained medically unstable and died, 137 were transferred to other departments, and 1829 became medically stable. Overall, we excluded 1497 of the 1829 patients. Of the remaining 332 patients we randomised 141 to locality based community hospital care (intervention group) and 79 to usual care (control group) (figure). Twenty six patients did not achieve group allocation: 16 in the community hospital group remained in the district general hospital and 10 in the usual care group were transferred to the community hospital. The main reasons for deviation from allocation were bed closures in the community hospital through infective gastroenteritis $(8 / 16)$ and extreme pressures on beds in the district general hospital $(8 / 10)$. In the community hospital group, 73 patients were transferred or died within two days of randomisation (72 transferred, one died), and 52 patients were transferred after more than two days or died in hospital after more than two days without transfer (49 transferred, three died). Eleven control patients were subsequently transferred to a non-locality based community hospital.

\section{Participant flow and follow up}

Both groups were well matched for baseline characteristics and length of hospital stay (table 1). Thirty two patients were not assessed one week after hospital discharge (29 died), 21 patients were not assessed at three months, and a further 13 patients were not assessed at six months (figure). Tables 2 and 3 provide the results of outcome measures. The outcome assessor was unblinded to treatment allocation at the six month assessment by 18 patients and carers in the treatment group and six patients and carers in the control group. The assessor correctly guessed the allocation of 67 of the remaining 128 patients (52\%) at the six month assessment (eight patients had missing data). Agreement was poor $(\kappa<0.20)$.

\section{Primary outcome analyses}

The unadjusted intention to treat comparison of changes in scores on the Nottingham extended activities of daily living scale at six months showed a non-significant difference between the groups (mean difference $4.88,95 \%$ confidence interval 0.18 to 9.59). A significant difference was suggested by the $t$ tests, but this was largely due to the effect of an outlier. The adjusted intention to treat comparison showed a significant difference between the two groups (mean difference 5.30, 0.64 to 9.96). Although the data were reasonably normal, we did find an outlier, which once

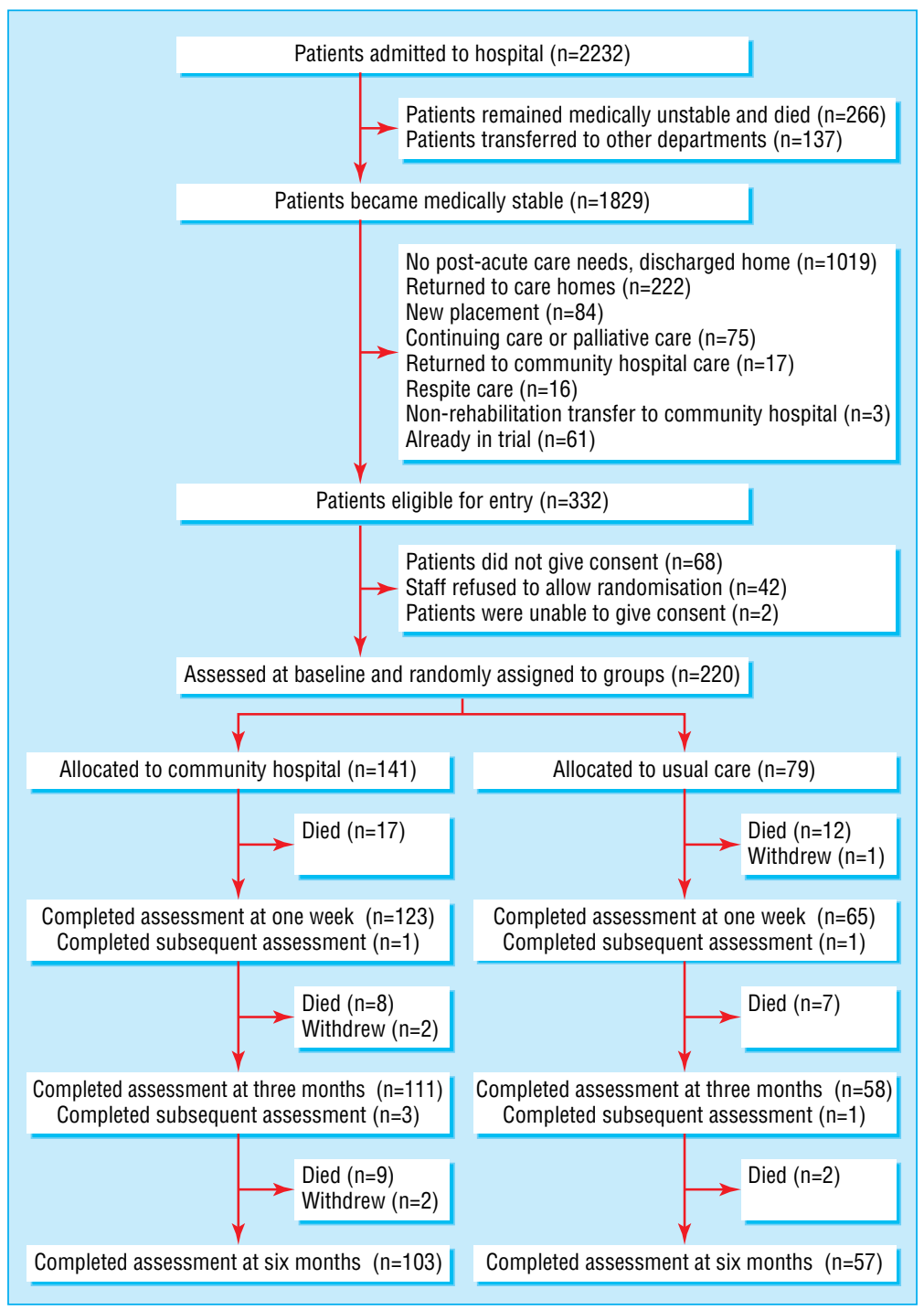

Flow of participants through trial 
Table 2 Summary scores for Nottingham extended activities of daily living scale at each assessment point

Community hospital group $(n=141)$

District general hospital group $(\mathrm{n}=79)$

\begin{tabular}{|c|c|c|c|c|c|c|}
\hline \multirow[b]{2}{*}{$\begin{array}{l}\text { Nottingham extended activities of } \\
\text { daily living scale }\end{array}$} & & \\
\hline & No of participants & Median (interquartile range) & Mean (SD) & No of participants & $\begin{array}{l}\text { Median (interquartile } \\
\text { range) }\end{array}$ & Mean (SD) \\
\hline \multicolumn{7}{|l|}{ Mobility: } \\
\hline Preadmission & 140 & $6(1-12)$ & $6.6(5.8)$ & 79 & $6(2-12)$ & $7.3(5.8)$ \\
\hline 3 months post-recruitment & 111 & $2(0-6)$ & $3.8(4.6)$ & 58 & $2(0-6)$ & $3.9(5.0)$ \\
\hline 6 months post-recruitment & 103 & $2(1-5)$ & $4.0(4.9)$ & 57 & $3(0-6)$ & $3.9(4.7)$ \\
\hline \multicolumn{7}{|l|}{ Kitchen: } \\
\hline 1 week post-discharge & 123 & $8(3-12)$ & $7.8(5.4)$ & 65 & $3(3-12)$ & $6.7(5.4)$ \\
\hline 3 months post-recruitment & 111 & $10(3-15)$ & $8.4(5.7)$ & 58 & $9(3-13)$ & $8.0(5.5)$ \\
\hline 6 months post-recruitment & 103 & $10(3-15)$ & $8.8(5.7)$ & 57 & $9(2-14)$ & $7.8(5.8)$ \\
\hline \multicolumn{7}{|l|}{ Domestic: } \\
\hline Preadmission & 140 & $6(3-10)$ & $6.2(4.8)$ & 79 & $6(3-11)$ & $6.8(4.5)$ \\
\hline 1 week post-discharge & 123 & $3(0-6)$ & $3.5(3.4)$ & 65 & $3(0-5)$ & $3.2(3.5)$ \\
\hline 3 months post-recruitment & 111 & $3(1-7)$ & $4.4(3.9)$ & 58 & $3(0-6)$ & $3.9(4.2)$ \\
\hline Preadmission & 140 & $6(4-9)$ & $7.0(3.9)$ & 79 & $7(3-10)$ & $7.4(3.9)$ \\
\hline 1 week post-discharge & 123 & $5(3-6)$ & $4.8(2.9)$ & 65 & $3(2-6)$ & $4.4(3.0)$ \\
\hline 3 months post-recruitment & 111 & $6(3-7)$ & $5.3(3.3)$ & 58 & $6(3-7)$ & $4.9(3.3)$ \\
\hline 6 months post-recruitment & 103 & $6(3-8)$ & $5.6(3.3)$ & 57 & $6(3-9)$ & $5.2(3.7)$ \\
\hline \multicolumn{7}{|l|}{ Total: } \\
\hline Preadmission & 140 & 32 (18-43) & $31.0(15.8)$ & 79 & $36(21-46)$ & 33.4 (15.3) \\
\hline 1 week post-discharge & 123 & $17(9-27)$ & $18.2(12.2)$ & 65 & $13(6-27)$ & $16.8(13.5)$ \\
\hline 3 months post-recruitment & 111 & $21(7-32)$ & $21.8(15.0)$ & 58 & $17(7-34)$ & $20.7(16.3)$ \\
\hline 6 months post-recruitment & 103 & $23(11-33)$ & $23.0(15.2)$ & 57 & $21(7-32)$ & $20.9(15.8)$ \\
\hline
\end{tabular}

removed had no material effect on the result (mean difference 4.68, 0.27 to 9.09). The difference remained after patients who had died were assigned the worst score on the Nottingham extended activities of daily living scale.

We found no differences between the groups for emotional distress in carers, measured using the general health questionnaire.

\section{Secondary analyses}

The secondary analyses showed few differences. We found improvements in favour of the community hospital in the sleep subsection of the Nottingham health profile at one week and three months (median difference $-10.5,-16.8$ to 0.0 and $-12.6,-22.4$ to 0.0 , respectively). The intervention group showed a greater increase in the depression subsection of the hospital anxiety and depression scale at one week (median difference 1.0, 0.0 to 2.0). Similar numbers of patients in the intervention and control groups died before the six month assessment (34 (23\%) $v 21$ (27\%), respectively) or were discharged to a new care home place (30/134 (22\%) v 21/74 (28.4\%), respectively).

Both groups had similar reported patient and carer satisfactions. At three months the intervention group showed greater satisfaction with the question "I get all the support I need from services such as meals on wheels, home helps, district nursing, etc" (odds ratio 3.43, 95\% confidence interval 1.05 to 11.24).

\section{Discussion}

We compared a locality based community hospital with predominantly extended stay in an elderly care department-a type of service for which there is reliable evidence that medium term outcomes are optimised. ${ }^{23}$ Transfer of older people to the community hospital for post-acute care was associated with greater functional independence, shown by the Nottingham extended activities of daily living scale change scores measured at six months after recruitment. The adjusted mean difference between the groups of over five points represents greater independence in at least two and possibly five of the 22 scale items-changes likely to be useful to patients and carers.

The study population will be familiar to practitioners working with older people: average age 85 , predominantly female, community dwelling, reduced independence before admission, and in receipt of care from social services. The participants had all presented as emergency admissions to hospital and were considered by their consultant to be medically stable and in need of additional rehabilitation before discharge home. This group of patients is a key focus for the new intermediate care services currently being established in England.

We were unable to find evidence of any benefit or detriment for the burden of carers as assessed by two quantitative measures. This finding should be contrasted with hospital at home care, another common post-acute service for older people for which there is concern about undue strain on carers. ${ }^{24}$ The main limitation to these conclusions relates to the modest sample sizes and especially carer groups and is reflected in the wide confidence limits for the estimates of effectiveness. A risk of bias also exists from the low response rate from carers. Both groups were similar for mortality, destination after discharge, length of hospital stay, and patient and carer satisfaction with services. The sleep section of the Nottingham health profile (but no other sections) showed a small difference in favour of the community hospital, and the depression subsection of the hospital anxiety and depression scale score showed a small difference in favour of the district general hospital. These results should be interpreted with caution, however, because of the small differences and because several comparisons were tested.

The results of our study should be interpreted against some important factors. Firstly, the study took place in a busy depart- 
Table 3 Summary scores of outcome measures for patients and carers at each assessment point

Community hospital group ( $n=141$ ) No of participants

Outcome measures

Patients: Barthel index

Baseline

1 week post-discharge

3 months post-recruitment

6 months post-recruitment

Patients: Nottingham health profile

Energy:

Baseline

1 week post-discharge

3 months post-recruitment

6 months post-recruitment

Pain:

Baseline

1 week post-discharge

3 months post-recruitment

6 months post-recruitment

Emotion:

Baseline

1 week post-discharge

3 months post-recruitment

6 months post-recruitment

Sleep:

Baseline

1 week post-discharge

3 months post-recruitment

6 months post-recruitment

Isolation:

Baseline

1 week post-discharge

3 months post-recruitment

6 months post-recruitment

Patients: Hospital anxiety and depression scale

Anxiety:

Baseline

1 week post-discharg

3 months post-recruitment

6 months post-recruitment

Depression:

Baseline

1 week post-discharge

3 months post-recruitment

6 months post-recruitment

Carers: General health questionnaire 28

Somatic symptoms:

Baseline

1 week post-discharge

3 months post-recruitment

6 months post-recruitment

Anxiety and insomnia

Baseline

1 week post-discharge

3 months post-recruitment

6 months post-recruitment

Social dystunction:

Baseline

1 week post-discharge

3 months post-recruitment

6 months post-recruitment

Severe depression:

Baseline

1 week post-discharge

3 months post-recruitment

6 months post-recruitment

Total:

Baseline

1 week post-discharge

3 months post-recruitment

6 months post-recruitment

Carer strain index:

1 week post-discharge

3 months post-recruitment

6 months post-recruitment
Median (interquartile range)

\begin{tabular}{|l}
$13(11-15)$ \\
\hline $16(13-18)$ \\
\hline $16(13-18)$ \\
\hline $17(14-19)$
\end{tabular}

16 (13-18)

$17(14-19)$

$61(24-100)$

$61(24-100)$

$37(0-100)$

$30(0-100)$

$19(0-45)$

$13(0-36)$

$17(0-43)$

$20(7-45)$

$15(0-44)$

$14(0-42)$

$14(0-28)$

$22(0-73)$

$22(0-51)$

$29(0-62)$

$22(0-73)$

$22(0-23)$

$19(0-23)$

$22(0-42)$

$0(0-23)$

$4(2-7)$

$5(1-8)$

$5(1-7)$

$4(2-7)$

$2(3-7)$

$5(3-9)$

$6(3-9)$

$6(3-9)$

$4(2-6)$

$3(2-7)$

$3(2-6)$

$5(1-7)$

$4(3-11)$

$5(1-10)$

$6(2-8)$

$5(1-8)$

$7(6-9)$

$7(5-7)$

$7(5-7)$

$0(0-3)$

$0(0-3)$

$0(0-3)$

$0(0-1)$

$1(0-8)$

$1(0-9)$

$3(0-5)$

$3(2-7)$

$3(1-8)$

$1(0-6)$
District general hospital group ( $\mathrm{n}=79$ ) No of participants

Median (interquartile range)

$\begin{array}{ll}79 & 13(11-1) \\ 65 & 16(14-1)) \\ 58 & 17(14-1)) \\ 57 & 16(13-1)\end{array}$




\section{What is already known on this topic}

Community hospitals are a long established component of healthcare provision in England

The contraction of the district general hospital and the shift towards locality based services have stimulated new developments in community hospitals

\section{What this study adds}

A locality based community hospital for post-acute care of older people was associated with greater functional independence

ment for the care of elderly people, with consequential operational difficulties for research: some staff refused access to eligible patients $(n=42)$, some patients were not treated as randomised $(n=26)$ owing to pressures on beds and temporary closures, and some patients experienced a delay in transfer to the community hospital. The deviations from treatment allocation may have introduced bias, although the number of patients was small in relation to the whole study group. Secondly, these are the results from a single centre and a single community hospital and therefore need to be confirmed by further studies. The results are potentially generalisable because of the similar median length of stay ( 15 days) between the community hospital in our study and other community hospitals. ${ }^{1}$ Lastly, the community hospital provided a locality based model of care. This is one of several possible care models offered by community hospitals ${ }^{1}$ in which the hospital is essentially an extension of primary care. Extrapolation to other community hospital care models, particularly one operating as an extension of secondary care, is speculative.

We thank our colleagues in the elderly care department, Bradford Teaching Hospitals NHS Foundation Trust; the managers and staff at Shipley community hospital; the North Bradford Primary Care Trust case management team; colleagues in the following organisations involved in the study: North Bradford Primary Care Trust, Bradford Community NHS Trust, Bradford Health Authority, Bradford social services department, University of Bradford, University of York Health Economics Consortium. The grant application signatories were JY, NS, KL, AF, and Angela Clegg. The trial steering group and project group members were JY, AF, NS, Susan Ince, Laura Hibbs, Angie Clegg, Joy Warburton, Jackie Hansford, Anne McAdam, KM, KL, Jacqueline O'Reilly, and JG. The study research team included Linda Dobrzanska, Helen Wright, Emma Tanner, KM, and JG.

Contributors: JG managed the study and was involved in data collection, collation and analysis, drafting the paper, and intellectual content. JY conceived the study, obtained funding, and was responsible for the design, initiation, and overall coordination of the study, analysis and interpretation of data, drafting the paper, and intellectual content; he is guarantor for the paper. AF obtained funding and was responsible for the design, initiation, and overall coordination of the study, interpretation of data, drafting the paper, and intellectual content. KM was involved in the initiation of the study, recruited patients, and was involved in writing the paper and its intellectual content. SB provided statistical input to the design of data analysis and interpretation of data and was involved in writing the paper and its intellectual content. KL contributed to the study design, obtained funding, and was responsible for the health economic component of the study. NS contributed to the study design, obtained funding, and was responsible for the qualitative components of the study.

Funding: Health Foundation.

Competing interests: JY and JG have worked in the community hospital involved in this study.

Ethical approval: Bradford Hospitals NHS Foundation Trust research ethics committee

1 Young J, Donaldson K. Community hospitals and older people. Age Ageing 2001;30-S3:7-10.

2 Bevan A. National Health Services Bill. House of Commons official report (Hansard). 30 Apr 1946:cols 43-149.

3 Department of Health. The NHS plan: a plan for investment, a plan for reform. Cm 4818-I. London: Stationery Office, 2000.

4 McCormack B. The developing role of community hospitals: an essential part of a quality service. Oual Saf Health Care 1993;2:253-8.

5 Baker JE, Goldacre M, Gray JAM. Community hospitals in Oxfordshire: their effect on the use of specialist inpatient services. J Epidemiol Community Health 1986;40:117-20.

6 Hine C, Wood VA, Taylor S, Charny M. Do community hospitals reduce the use of district general hospital inpatient beds? J R Soc Med 1996;89:681-7.

7 Cook PJ, Porter L. Community hospitals and district general hospital medical bed use by elderly people: a study of 342 general practitioner beds in Oxfordshire. Age Ageing 1998;27:357-61.

8 Department of Health. National service framework for older people. London: DoH, 2001.

9 Department of Health. Intermediate care. London: DoH, 2001. (HSC 2001/01: LAC (2001) 01.)

10 Brook RH, Kahn KL, Kosecoff J. Assessing clinical stability at discharge. JAMA 1992;268:1321-2.

11 Hodkinson HM. Evaluation of a mental test score for assessment of mental impairment in the elderly. Age Ageing 1972;1:233-8.

12 Nouri FM, Lincoln NB. An extended activities of daily living scale for stroke patients. Clin Rehabil 1987:1:301-5.

13 Mahoney FI, Barthel DW. Functional evaluation: the Barthel index. Md State Med J 1965;14:61-5

14 Collin C, Wade DT, Davies S, Horne V. The Barthel ADL index: a reliability study. Int Disabil Stud 1988;10:61-3.

15 Harwood RH, Ebrahim S. The validity, reliability and responsiveness of the Nottingham extended activities of daily living scale in patients undergoing total hip replacement. Disabil Rehabil 2002;24:371-7.

16 Goldberg DP, Hillier VF. A scaled version of the general health questionnaire. Psychol Med 1979;9:139-45.

17 Hunt SM, McEwen J, McKenna SP. Measuring health status. London: Croom Helm, 1986.

18 Zigmond AS, Snaith RP. The hospital anxiety and depression scale. Acta Psychiatr Scand 1983;67:361-70.

19 Pound P, Gompertz P, Ebrahim S. Patients' satisfaction with stroke services. Clin Rehabil 1994:8:7-17.

20 Robinson BC. Validation of a caregiver strain index.J Gerontol 1983;38:344-8.

21 Pound P, Gompertz P, Ebrahim S. Development and results of a questionnaire to measure carer satisfaction after stroke. J Epidemiol Community Health 1993;47:500-5.

22 Young J, Robinson M, Chell S, Sanderson D, Chaplin S, Burns E, et al. A prospective baseline study of frail older people before the introduction of an intermediate care service. Health Soc Care Community, 2005; 13:307-12.

23 Stuck AE, Siu AL, Wieland GD, Adams J, Rubenstein LZ. Comprehensive geriatric assessment: a meta-analysis of controlled trials. Lancet 1993;342:1032-6.

24 Shepperd S, Iliffe S. Hospital at home versus in-patient hospital care. Cochrane Database Syst Rev 2001;(2):CD000356.

(Accepted 15 May 2005)

doi 10.1136/bmj.38498.387569.8F

Department of Health Care for the Elderly, St Luke's Hospital, Bradford BD5 0NA John Green research fellow

John Young consultant geriatrician

Anne Forster principal research fellow

Karen Mallinder research nurse

Aysgarth Statistics, Beaconsfield HP9 2AS

Sue Bogle consultant statistician

York Health Economics Consortium, University of York, York YO10 5NH

Karin Lowson project director

School of Health Studies, University of Bradford, Bradford BD5 0BB

Neil Small professor of health research

Correspondence to:J Green becarrd.1@bradfordhospitals.nhs.uk 